\title{
Direct observation of ultrafast plasmonic hot electron transfer in the strong coupling regime
}

\author{
Hangyong Shan $\mathbb{D}^{1}$, Ying Yu', Xingli Wang ${ }^{2}$, Yang Luo', Shuai Zu', Bowen Du', Tianyang Han ${ }^{1}$, Bowen Li ${ }^{1}, Y u \mathrm{Li}^{1}$,

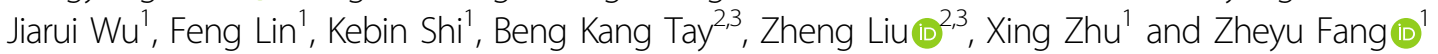

\begin{abstract}
Achieving strong coupling between plasmonic oscillators can significantly modulate their intrinsic optical properties. Here, we report the direct observation of ultrafast plasmonic hot electron transfer from an Au grating array to an $\mathrm{MoS}_{2}$ monolayer in the strong coupling regime between localized surface plasmons (LSPs) and surface plasmon polaritons (SPPs). By means of femtosecond pump-probe spectroscopy, the measured hot electron transfer time is approximately $40 \mathrm{fs}$ with a maximum external quantum yield of $1.65 \%$. Our results suggest that strong coupling between LSPs and SPPs has synergetic effects on the generation of plasmonic hot carriers, where SPPs with a unique nonradiative feature can act as an 'energy recycle bin' to reuse the radiative energy of LSPs and contribute to hot carrier generation. Coherent energy exchange between plasmonic modes in the strong coupling regime can further enhance the vertical electric field and promote the transfer of hot electrons between the Au grating and the $\mathrm{MoS}_{2}$ monolayer. Our proposed plasmonic strong coupling configuration overcomes the challenge associated with utilizing hot carriers and is instructive in terms of improving the performance of plasmonic opto-electronic devices.
\end{abstract}

\section{Introduction}

Surface plasmons (SPs), as the collective oscillation of free electrons at the interface between dielectric and metal layers ${ }^{1}$, have aroused tremendous interest in diverse fields, such as solar energy conversion, superresolution, high harmonic generation, near-field imaging, and nonlinear phenomena $^{2-9}$. As nonpropagating SPs, localized surface plasmons (LSPs) can either dephase radiatively by reemitting photons or decay by Landau damping to form energetic electron-hole pairs ${ }^{10,11}$. These pairs are nonthermal, and their intense collisions can redistribute accumulated energy in hundreds of femtoseconds,

Correspondence: Zheyu Fang (zhyfang@pku.edu.cn)

'School of Physics, State Key Lab for Mesoscopic Physics; Academy for Advanced Interdisciplinary Studies; Collaborative Innovation Center of

Quantum Matter, Peking University, 100871 Beijing, China

${ }^{2}$ CNRS International-NTU-Thales Research Alliance (CINTRA), Nanyang

Technological University, Singapore 637553, Singapore

Full list of author information is available at the end of the article.

These authors contributed equally: Hangyong Shan, Ying Yu. developing into hot carriers that obey a Fermi-Dirac-like distribution with an increased effective temperature ${ }^{2,3}$. If these hot carriers are exported at a rate faster than energy dissipation by electron-phonon scattering, they can be collected and utilized in external circuits for optoelectronic devices such as photodetectors ${ }^{12-26}$. To realize this application, two critical challenges must be overcome: the large radiative rate of LSPs and the rapid relaxation of the hot carriers.

In contrast to LSPs, surface plasmon polaritons (SPPs) relax almost nonradiatively even at rough surfaces, leading to a higher photon-to-carrier conversion efficiency $^{27,28}$. However, such carriers with lower energies have a low probability of crossing the potential barrier between a metal and semiconductor, which leads to a low output yield in practice $^{29}$. In addition, the lack of vertical momentum and interfacial reflection also block the exportation of SPP-generated carriers ${ }^{29}$. Photons

\section{(c) The Author(s) 2019}

(c) (i) Open Access This article is licensed under a Creative Commons Attribution 4.0 International License, which permits use, sharing, adaptation, distribution and reproduction c. in any medium or format, as long as you give appropriate credit to the original author(s) and the source, provide a link to the Creative Commons license, and indicate if changes were made. The images or other third party material in this article are included in the article's Creative Commons license, unless indicated otherwise in a credit line to the material. If material is not included in the article's Creative Commons license and your intended use is not permitted by statutory regulation or exceeds the permitted use, you will need to obtain permission directly from the copyright holder. To view a copy of this license, visit http://creativecommons.org/licenses/by/4.0/. 
absorbed by SPPs are hence mostly exhausted as heat rather than transformed into exploitable electrical energy.

Here, we consider that the distinct properties of LSPs and SPPs may synergize to produce plasmonic hot carriers. In the weak coupling regime, the interaction between two oscillators only introduces a perturbation to their original properties; thus, SPPs exert little influence on the intrinsic radiative damping of LSPs. However, the energy levels of hybrid polaritons can be greatly altered when oscillators strongly interact with each other in a phenomenon called strong coupling, in which Rabi splitting can be experimentally observed as a distinguishable characteristic of the energy spectrum ${ }^{30-36}$. When the coupling strength exceeds the decoherence rate of the original oscillator, strong coupling occurs ${ }^{30}$, and the energy exchange between the oscillators becomes the dominant relaxation channel. It has been proven that the resonant radiative rate of harmonic oscillators can be modulated in the strong coupling regime ${ }^{37-39}$, which is conducive to addressing the radiation damping bottleneck in the exploitation of hot carriers decayed from $\mathrm{LSPs}^{37}$.

In this article, we propose a metal-insulator-metal (MIM) sandwiched heterostructure, where an $\mathrm{MoS}_{2}$ monolayer is employed to constitute a Schottky heterojunction with an Au grating on top and serves as an acceptor to harvest hot electrons that decay from LSPs. Stemming from the strong coupling between LSPs and SPPs, hot electron transfer at this heterojunction can be facilitated by coherent energy exchange and the perpendicular enhanced electric field, which decreases the radiative rate of the LSPs and accelerates the exportation of hot electrons. The physical insight presented in this work paves the way to construct plasmonic hot carrier devices with improved performance in the future.

\section{Results}

Figure 1a shows a schematic of our Au grating/ $\mathrm{MoS}_{2} /$ substrate sandwiched structure, where the substrate consists of a $20 \mathrm{~nm} \mathrm{Al}_{2} \mathrm{O}_{3}$ spacer and a $50 \mathrm{~nm}$ Au layer evaporated on a $\mathrm{Si} / \mathrm{SiO}_{2}$ wafer. Scanning electron microscopy (SEM) images of the heterostructures with different grating periods are shown in Fig. $1 \mathrm{~b}$ and Figure S1. The electronic band alignment diagram of the Au grating and $\mathrm{MoS}_{2}$ monolayer is illustrated in Fig. 1c, which also sketches the transfer process of the plasmonic hot electrons. These electrons are excited by a $780 \mathrm{~nm}$ pump laser. After crossing the Schottky barrier, they are injected into the $\mathrm{MoS}_{2}$ monolayer underneath and induce a variation in the filled states therein, which is monitored by a $650 \mathrm{~nm}$ probe pulse. In the experiment, the $20 \mathrm{~nm} \mathrm{Al}_{2} \mathrm{O}_{3}$ layer can prevent hot carriers that decay from SPPs from tunneling into the $\mathrm{MoS}_{2}$ monolayer, although most carriers are distributed in the low-energy region and can hardly cross the Schottky barrier. As the energy of the pump laser $(1.59 \mathrm{eV})$ is lower than the bandgap of the $\mathrm{MoS}_{2}$ monolayer, excitons cannot be directly excited. Therefore, it is safe to consider that the detected transient absorption signal is only induced by the injection of hot electrons that decay from LSPs, indicating the direct observation of plasmonic hot electron transfer.

Figure 1d shows plots of the measured reflectance spectra of a pristine $\mathrm{MoS}_{2}$ monolayer, bare Au grating array and hybrid $\mathrm{Au}$ grating/ $\mathrm{MoS}_{2}$ structure at the substrate. Two absorption peaks were observed at $605 \mathrm{~nm}(\boldsymbol{B}$ exciton) and $654 \mathrm{~nm}$ ( $\boldsymbol{A}$ exciton) for the pristine $\mathrm{MoS}_{2}$ monolayer (olive line). Regarding the bare Au grating (magenta line), three obvious resonances appeared at 615, 725 , and $823 \mathrm{~nm}$. For the hybrid Au grating/ $\mathrm{MoS}_{2}$ structure (orange line), the third resonance peak was redshifted by approximately $25 \mathrm{~nm}$ due to the change in the surrounding dielectric medium. From the above results, we can see that the third resonance is primarily due to LSPs, while the first two resonances mainly result from $\mathrm{SPPs}^{40}$. In fact, all of these resonances are coupled polaritons that hybridize between LSPs and SPPs along either the $-x$ $\left(\mathrm{SPP}_{1}\right)$ or $+x\left(\mathrm{SPP}_{2}\right)$ axes (Figure $\left.\mathrm{S} 2\right)$. In Fig. $1 \mathrm{~d}$, the dashed lines are FDTD simulation results for the bare $\mathrm{Au}$ grating array and $\mathrm{Au} / \mathrm{MoS}_{2}$ hybrid structure, which are in good agreement with the experimental results (see Materials and methods for details).

It is well known that a steady-state reflectivity measurement is an efficient way to investigate strong coupling. Figure $2 \mathrm{a}, \mathrm{b}$ shows the measured and simulated reflectance spectra of $\mathrm{Au} / \mathrm{MoS}_{2}$ hybrids with Au grating periods ranging from 600 to $750 \mathrm{~nm}$, where the three plasmon resonances behave in a similar fashion. The magnetic field distribution of these resonances for a grating period of $700 \mathrm{~nm}$ was calculated in Fig. 2c. It is apparent that the mode at $704 \mathrm{~nm}(798 \mathrm{~nm})$ is dominated by SPPs propagating along the $+x(-x)$ axis, and the third resonance is mainly due to LSPs. Similar results can be obtained from the electric field distribution in Figure S3 and Supplementary Section 1.

Figure $2 \mathrm{~d}$ shows the simulated reflectance mapping for grating periods ranging from 550 to $1000 \mathrm{~nm}$, where two clear energy anticrossings appear. These avoided crossings correspond to Rabi splitting and demonstrate strong coupling between the uncoupled modes. A coupled oscillator model was successfully used to study the observed strong coupling ${ }^{41-43}$. In our case, the Hamiltonian $H$ can be written as

$$
\left(\begin{array}{ccc}
E_{L S P s} & V_{1} & V_{2} \\
V_{1}^{*} & E_{S P P_{1}} & V_{3} \\
V_{2}^{*} & V_{3}^{*} & E_{S P P_{2}}
\end{array}\right)
$$

where $E_{\mathrm{LSP}}, E_{\mathrm{SPP} 1}$ and $E_{\mathrm{SPP} 2}$ are the resonance energies of the uncoupled LSP, $\mathrm{SPP}_{1}$, and $\mathrm{SPP}_{2}$ states, and $V_{i}(i=1,2$, 

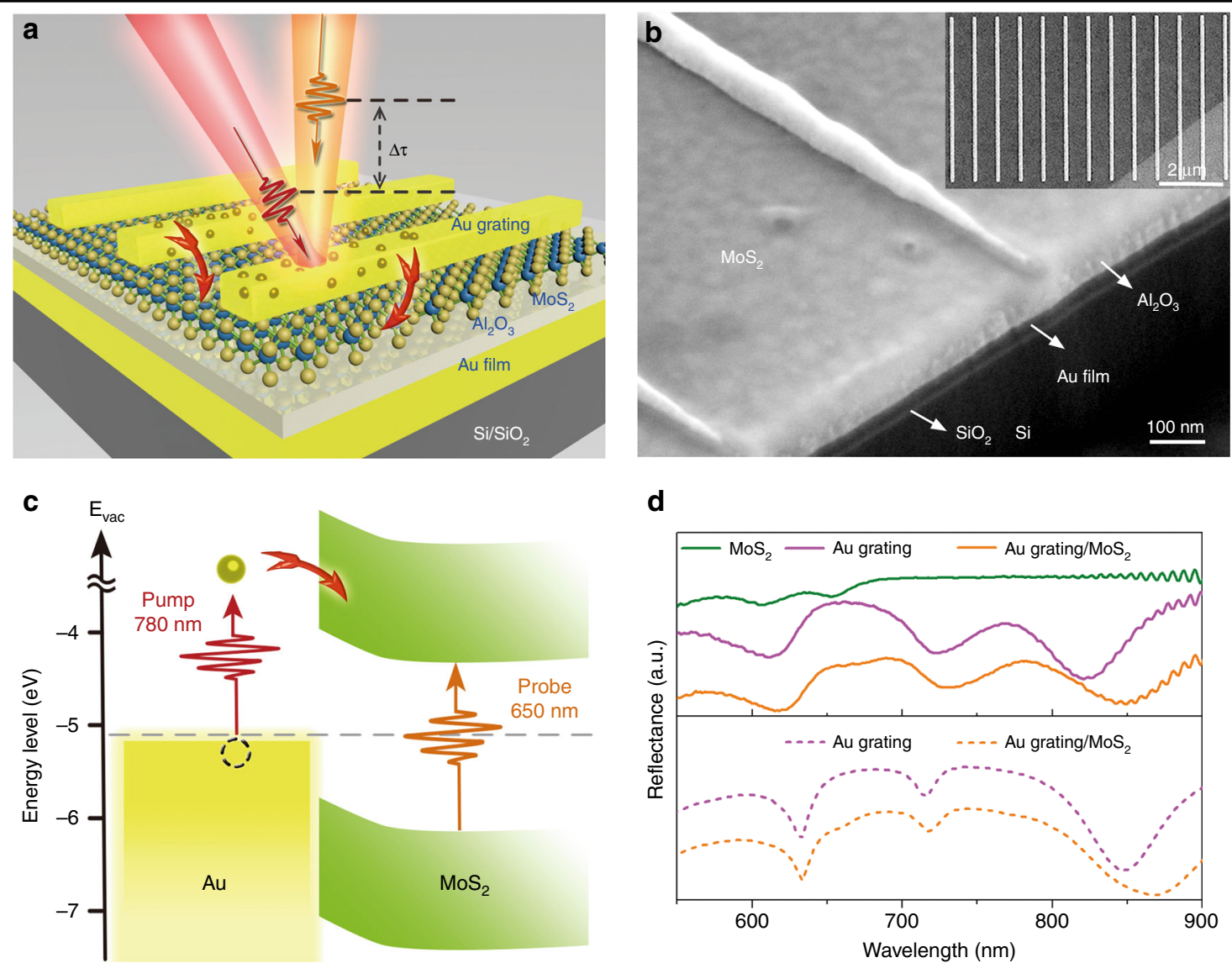

Fig. 1 Characterizations of the heterostructure, including an SEM image, a band alignment diagram and reflectance spectra. a Schematic of the $\mathrm{Au}$ grating $/ \mathrm{MoS}_{2} / \mathrm{Al}_{2} \mathrm{O}_{3} / \mathrm{Au} / \mathrm{Si}$ sandwiched heterostructure. $\mathbf{b}$ Cross-sectional SEM view of a typical MIM heterostructure, imaged at a $52^{\circ}$ tilted angle. The inset shows a top-view SEM image for gratings with a $700 \pm 5 \mathrm{~nm}$ period. The gray area corresponds to the substrate, while the dark gray represents the $\mathrm{MoS}_{2}$ monolayer. c The band alignment diagram of the Au grating and $\mathrm{MoS}_{2}$ monolayer. Plasmonic hot electrons were pumped at $780 \mathrm{~nm}$, and the transient absorption of the $\boldsymbol{A}$ exciton in the $\mathrm{MoS}_{2}$ monolayer was monitored by a $650 \mathrm{~nm}$ probe pulse. $\mathbf{d}$ Experimentally measured reflectance spectra of the $\mathrm{MoS}_{2}$ monolayer, Au grating and Au grating/MoS 2 at the substrate with a $600 \pm 5 \mathrm{~nm}$ grating period (solid lines). Reflectance spectra with plasmonic structures were also simulated by the finite-difference time-domain (FDTD) method (dashed lines), which show good agreement with the measurements. SEM scanning electron microscopy

3) represent the coupling strengths between these states. $\Psi_{i}(i=1,2,3)$ are defined as eigenstates of $H$ with the lowest, middle, and highest eigenenergies. They are also mixtures of uncoupled modes and can be expressed as

$$
\Psi_{i}=c_{i 1}|L S P S\rangle+c_{i 2}\left|S P P_{1}\right\rangle+c_{i 3}\left|S P P_{2}\right\rangle
$$

where $c_{i j}(j=1,2,3)$ are coefficients.

To solve the Hamiltonian, the dispersion relations of the uncoupled LSPs, $\mathrm{SPP}_{1}$, and $\mathrm{SPP}_{2}$ are plotted in Fig. $2 \mathrm{e}$ as dotted, dashed, and dash-dotted lines, respectively, from which we can see that the LSP resonance energy $\left(E_{\mathrm{LSPs}}=1.46 \mathrm{eV}\right)$ remains constant as the grating period changes. For the uncoupled SPP modes, their dispersion relations can be obtained from the equation

$$
\pm K_{s p p}=K_{x} \pm m \frac{2 \pi}{P}
$$

where $K_{s p p}=\frac{\omega}{c} \sqrt{\frac{\varepsilon_{1} \varepsilon_{2}}{\varepsilon_{1}+\varepsilon_{2}}}$ is the SPP wavevector, $\varepsilon_{1}\left(\varepsilon_{2}\right)$ is the dielectric permittivity of the $\mathrm{Au}$ (dielectric) layer, $K_{x}=$ $\frac{\omega}{c} \sin \theta$ is the horizontal wavevector component of the incident light, $m$ is an integer and $P$ corresponds to the grating period. For such an air $/ \mathrm{Al}_{2} \mathrm{O}_{3} / \mathrm{Au}$ interface, the dispersion relation of the SPPs cannot be directly described by a classic model of two semi-infinite layers. However, the actual dispersion can be acquired from a modified model, in which an effective medium with a wavelength-dependent refractive index is introduced (Figure S4 and Supplementary Section 2).

With the fitting parameters $V_{i}(i=1,2,3)$ equal to $0.075,0.075$, and $0.06 \mathrm{eV}$, respectively, theoretical results based on the coupled oscillator model were calculated. The results are shown as red solid lines in Fig. 2e. $V_{1}=V_{2}$ means that the coupling strength between the LSPs and $\mathrm{SPP}_{1}$ equals that between the LSPs and $\mathrm{SPP}_{2}$, which may 
a
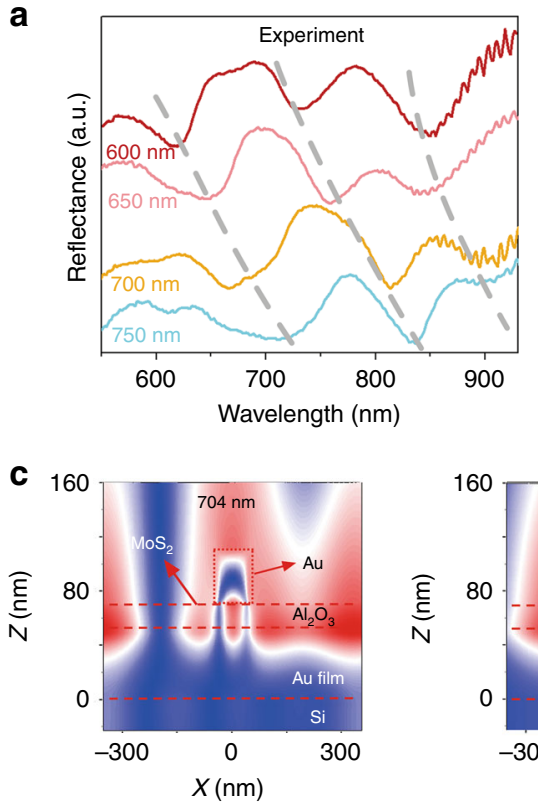

d

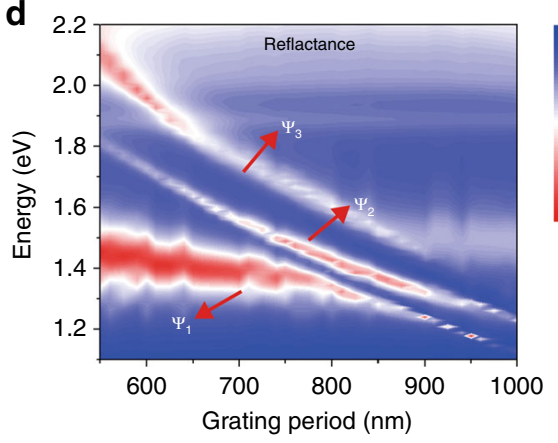

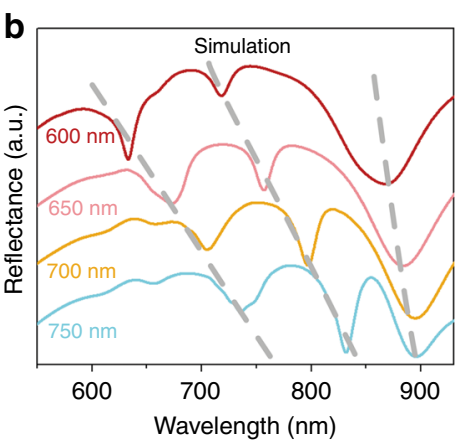
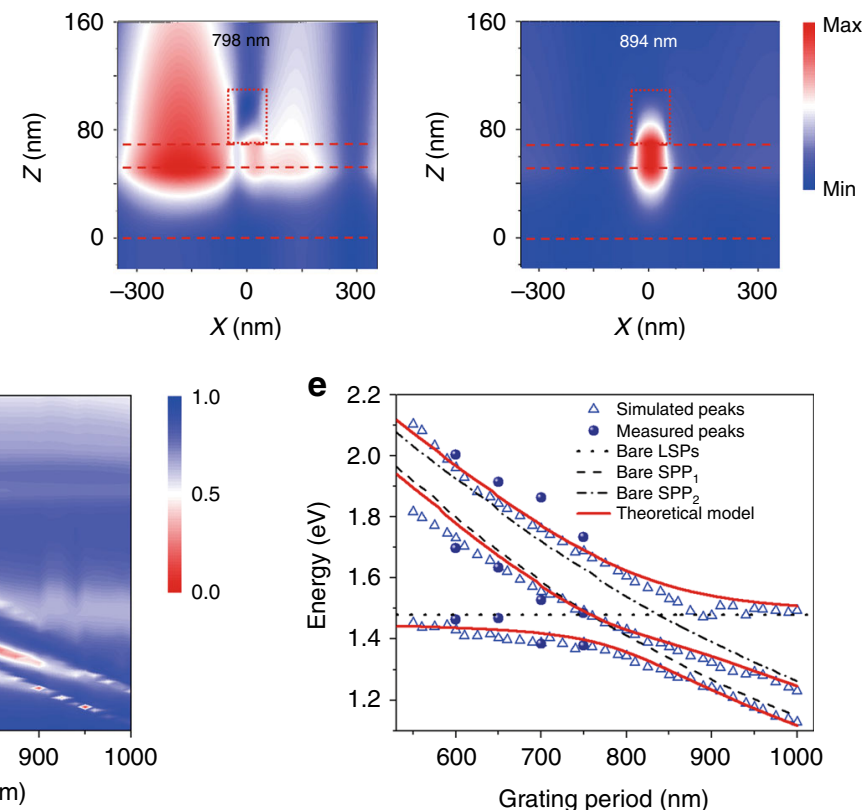

Fig. 2 Steady-state reflectance spectra of the sandwiched heterostructures. a Measured reflectance spectra of the Au grating/MoS $/$ substrate with grating periods of $600,650,700$, and $750 \mathrm{~nm}$. b Simulated reflectance spectra of the corresponding samples in (a). c Simulated magnetic field distribution at the resonances for the sample with a $700 \mathrm{~nm}$ grating period. $\mathbf{d}$ The simulated grating-period-dependent reflectance of the sandwiched structures, which exhibits classic Rabi splitting. $\Psi_{i}(i=1,2,3)$ are eigenstates of the Hamiltonian. e Simulated (hollow triangles), measured (filled dots), and theoretical (solid lines) resonance energies as a function of the grating period. The dotted line shows the constant dispersion relation of the uncoupled localized surface plasmons, while the dashed and dash-dotted lines denote the dispersions of the uncoupled SPPs propagating along the $-x\left(\mathrm{SPP}_{1}\right)$ and $+x\left(\mathrm{SPP}_{2}\right)$ axes, respectively. SPP surface plasmon polariton

be attributed to $m=1$ for both $\mathrm{SPP}_{1}$ and $\mathrm{SPP}_{2}$. The simulated (hollow triangles), measured (filled dots), and theoretical (solid lines) results are in excellent agreement, as demonstrated in Fig. 2e, which implies that strong coupling between the LSPs and SPPs occurs in our proposed MIM heterostructures.

To investigate the dynamics of plasmonic hot electron transfer in the strong coupling regime between the LSPs and SPPs, femtosecond pump-probe measurements in a reflection configuration were carried out. Figure 3a presents differential reflection spectra $\Delta R / R_{0}(\mathrm{t})$ of the heterostructure with a $700 \mathrm{~nm}$ Au grating period (red dots) and reference samples, which include a bare Au grating array on the substrate (blue), a pristine $\mathrm{MoS}_{2}$ monolayer on the substrate (green) and the substrate (gray). The pump had a wavelength of $780 \mathrm{~nm}$ and a fluence of $7.5 \mu \mathrm{J} /$ $\mathrm{cm}^{2}$, and $650 \mathrm{~nm}$ probe pulses with a fluence of $0.75 \mu \mathrm{J} /$ $\mathrm{cm}^{2}$ were used to detect the transient absorption of the $A$ exciton in the $\mathrm{MoS}_{2}$ monolayer. For the bare Au grating array on the substrate, no transient differential reflection signal appears, proving that plasmonic hot electron relaxation cannot induce ultrafast signals in this case. One of the causes of this phenomenon could be the low pump and probe fluences in our experiments, which are two orders of magnitude weaker than those used in typical pump-probe measurements of metallic electron relaxations ${ }^{44}$. This reason is verified in Figure S5 and Supplementary Section 3, in which the pump fluence is enhanced by two orders of magnitude. The other reason is the pump wavelength of $780 \mathrm{~nm}$ pump, which is chosen to excite SP 

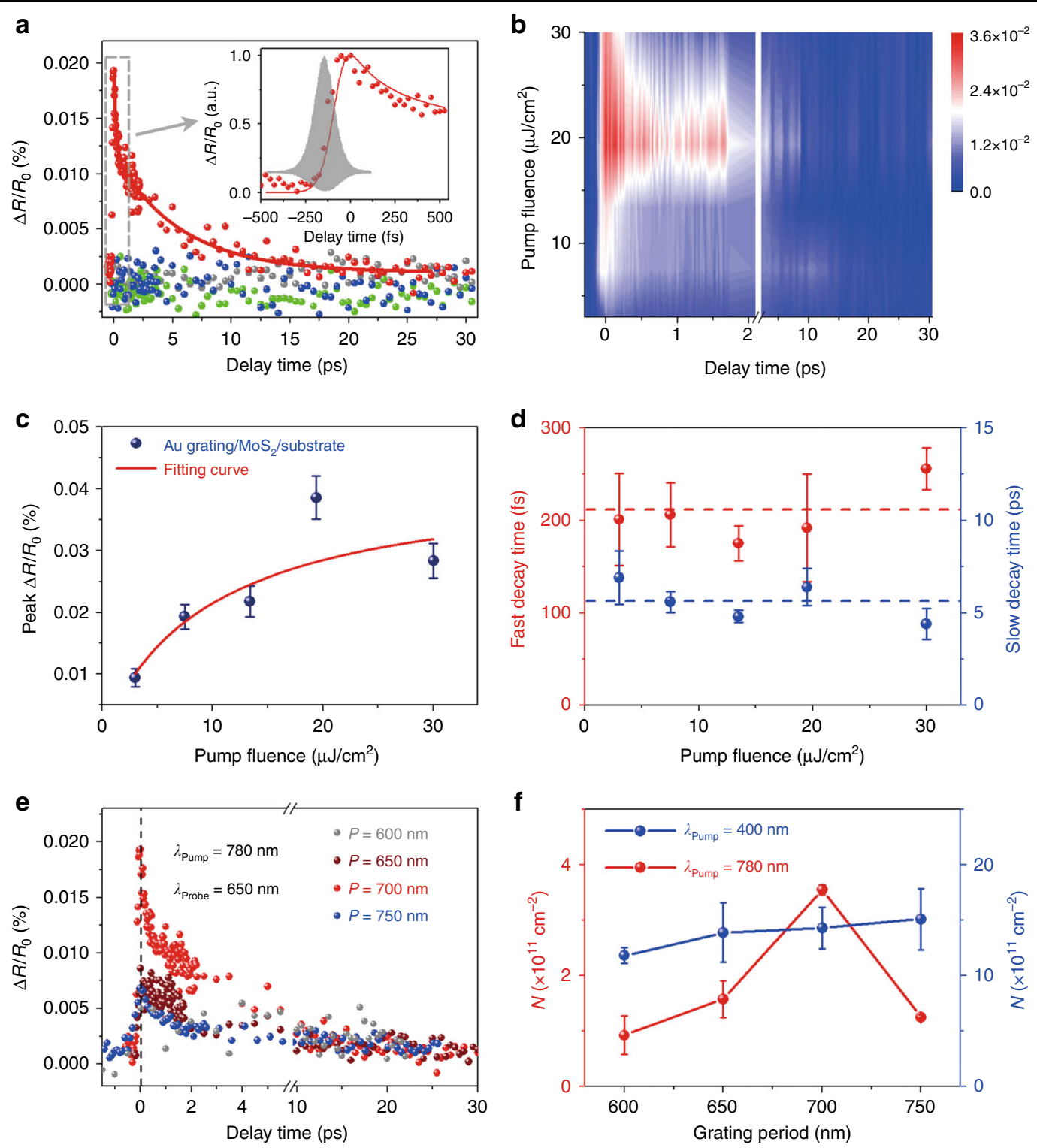

Fig. 3 Transient absorption spectra of the heterostructures for different grating periods and pump wavelengths. a Differential reflection spectra of the Au grating/MoS $/$ substrate (red dots) and control samples of the Au grating, $\mathrm{MoS}_{2}$ monolayer and substrate for a pump fluence of $7.5 \mu \mathrm{J} / \mathrm{cm}^{2}$. The red line is a fitting curve for the descent of $\Delta R / R_{0}(t)$ with a biexponential function. The inset shows details of the rising process with normalization. The data shown in gray represent the interferometric autocorrelation function for the pump pulses, from which the upper envelope with a full width at half-maximum of $\sim 130 \mathrm{fs}$ is taken as the IRF. The red line in the inset was obtained by convoluting the IRF and the actual electron injection function. $\mathbf{b}$ Pump-fluence-dependent transient absorption spectra of the heterostructure. The scale bar corresponds to the intensity of the ultrafast signals. $\mathbf{c}$ The maximum amplitude of $\Delta R / R_{0}(t)$ as a function of pump fluence. The red line is a fitting curve with the saturation formula of Eq. (4). $\mathbf{d}$ Fast and slow decay lifetimes varying with pump fluence. e Ultrafast pump-probe spectra of the Au grating/MoS $/$ substrate for different grating periods pumped at $780 \mathrm{~nm}$ with $7.5 \mathrm{\mu J} / \mathrm{cm}^{2}$. f Excited (injected) electron densities derived from the peak amplitudes of $\Delta R / R_{0}(t)$. The red dots represent the grating-period-dependent densities of electrons injected into the $\mathrm{MoS}_{2}$ monolayer pumped at $780 \mathrm{~nm}$, while the blue dots correspond to the densities of electrons excited directly at the pump wavelength of $400 \mathrm{~nm}$. IRF instrument response function

resonances rather than interband transitions of $\mathrm{Au}$ that have larger absorption cross-sections ${ }^{44}$.

The ultrafast signal only emerges in the Au grating/ $\mathrm{MoS}_{2} /$ substrate heterostructure, revealing that the variation in the occupancy in $\mathrm{MoS}_{2}$ arises from plasmonic hot electron injection. As the injected hot electrons fill unoccupied states in the conduction band of $\mathrm{MoS}_{2}$ and rapidly relax to the exciton level, the absorbance of the $\boldsymbol{A}$ exciton with the pump is lower than that without the pump. Thus, the transfer process can be represented by the rising edge of $\Delta R / R_{0}(t)$, as shown in the inset. The injection time was estimated to be approximately $40 \mathrm{fs}$ by 
deconvoluting the signal with the instrument response function (IRF) (see Supplementary Section 5 for the detailed deconvolution process). The red line in the inset was obtained by convoluting the IRF and the actual electron injection function, which can well reproduce the experimentally measured transient differential signal. Then, the decay of transferred hot electrons occurs during the descent of $\Delta R / R_{0}(t)$. The decay process can be well fitted with a biexponential function and is caused by electron-electron and electron-phonon scattering.

The pump fluence dependence of the ultrafast signal was studied in Fig. 3b, and the maximum amplitudes of the spectra were extracted in Fig. 3c. The peak amplitude increases and gradually saturates as the fluence increases, and the relationship can be fitted by

$$
\Delta R / R_{0}(0) \propto \frac{f}{f+f_{\text {sat }}}
$$

where $f$ and $f_{\text {sat }}$ are the pump fluence and the saturation value, respectively. When the fluence exceeds $10 \mu \mathrm{J} / \mathrm{cm}^{2}$, the peak of $\Delta R / R_{0}(t)$ is greatly influenced by the saturation effect. This phenomenon could be caused by a mass accumulation of transferred hot electrons because these electrons repel like charges and prevent further injection into the $\mathrm{MoS}_{2}$ monolayer. Different from the saturating trend of the peak amplitude, the decay processes in the transient absorption spectra are independent of the pump fluence, as shown in Fig. 3d, and can be fitted by biexponential functions with average parameters of $210 \mathrm{fs}$ and $5.5 \mathrm{ps}$, which are attributed to the lifetimes of electron -electron and electron-phonon interactions.

Apart from the transfer timescale, the injected hot electron density is another important factor. As ultrafast signals arise from variations in occupation number in the $\mathrm{MoS}_{2}$ monolayer, the largest intensity of $\Delta R / R_{0}(t)$ can in principle represent the density of excited (injected) electrons. At a pump wavelength of $780 \mathrm{~nm}$, ultrafast signals arise from the injection of plasmonic hot electrons. As a result, the densities of the electrons transferred into $\mathrm{MoS}_{2}$ cannot be directly evaluated by the absorbance of $\mathrm{MoS}_{2}$. To derive the densities in this case, a quantitative relationship between the densities and peak amplitudes of $\Delta R / R_{0}(\mathrm{t})$ needs to be established first. To obtain this relation, transient absorption spectra pumped at $400 \mathrm{~nm}$ were measured, because ultrafast signals originate from the direct excitation of electrons in the valence band of $\mathrm{MoS}_{2}$ in this case. Therefore, the excited electron density can be calculated from the absorbance of the $\mathrm{MoS}_{2}$ monolayer. At a pump wavelength of $400 \mathrm{~nm}$ with $7.5 \mu \mathrm{J} / \mathrm{cm}^{2}$, the excited electron density equals $1.47 \times 10^{12} \mathrm{~cm}^{-2}$ with a corresponding peak amplitude of $\Delta R / R_{0}(t)$ of $0.08 \%$ (Figure S6). Based on this result, a linear relationship between the density and peak amplitude was eventually established (see Supplementary Section 6 for details). The density of injected hot electrons pumped at $780 \mathrm{~nm}$, which was derived from the peak amplitude in Fig. 3a, was estimated as $3.55 \times 10^{11} \mathrm{~cm}^{-2}$.

Moreover, to further confirm the active role of SPs in injecting hot electrons, ultrafast transient absorption measurements of samples pumped at $780 \mathrm{~nm}$ for various grating periods were performed, as shown in Fig. 3e. Based on their peak amplitudes, the transferred electron densities were calculated similarly in Fig. 3f. The density reaches a maximum for a grating period of $700 \mathrm{~nm}$ because strong coupling occurs therein and the resonance peak of $\Psi_{2}$ (the eigenstate with the middle eigenenergy) is closer to the pump wavelength than the resonances of the other grating periods (Fig. 2a). In contrast, the excited electron density hardly changes with the grating period when the pump wavelength is $400 \mathrm{~nm}$, as illustrated in Fig. 3f and Figure S7, in which case the plasmonic modes cannot be resonantly excited. This distinct grating period dependence at different pump wavelengths is ascribed to the diverse origins of the occupancy number variation in $\mathrm{MoS}_{2}$.

From the above results, plasmonic hot electron transfer from the $\mathrm{Au}$ grating to the $\mathrm{MoS}_{2}$ monolayer was directly observed in the strong coupling regime, in which the injection time and density were approximately $40 \mathrm{fs}$ and $3.55 \times 10^{11} \mathrm{~cm}^{-2}$, respectively, for a pump fluence of $7.5 \mu \mathrm{J} / \mathrm{cm}^{2}$.

The injection efficiency is an important factor in carrier transfer studies. In our work, there is also significant evidence to prove the contribution of strong coupling in advancing plasmonic hot electron transfer. Here, we reveal the effect of strong coupling by comparing experimental and theoretical electron injection yields. The external quantum yield $\eta$ is defined as the ratio of transferred electron densities to incident photon densities. To obtain a maximum yield in the experiment, the pump wavelength was altered, as shown in Fig. 4a. The resonance wavelength of $\Psi_{2}$ is $813 \mathrm{~nm}$ for the $700 \mathrm{~nm}$ grating period, and the peak of $\eta$ follows the resonance, reaching its largest value of $1.65 \%$ at the pump wavelength of $810 \mathrm{~nm}$.

To estimate theoretical efficiencies, we first calculated all branches of $\Psi_{i}(i=1,2,3)\left(\left|c_{i j}\right|^{2}\right)$ for different grating periods in Fig. 4b and Figure S8 according to the coupled oscillator model. For a grating period of $700 \mathrm{~nm}$, the LSP branches $\left(\left|c_{i 1}\right|^{2}\right)$ are approximately 85,6 , and $10 \%$ in $\Psi_{i}$ $(i=1,2,3)$, respectively, in agreement with the magnetic field distribution in Fig. 2c. Since $\Psi_{2}$ is near-resonant with a $780 \mathrm{~nm}$ pump, its fractions are analyzed in detail in Fig. 4b. It is interesting to note that $\mathrm{SPP}_{1}$ dominates $\Psi_{2}$ and the LSPs only contribute $6 \%$ for a $700 \mathrm{~nm}$ grating period, which implies that the LSPs are launched with 6\% of the energy in $\Psi_{2}$.

Next, we evaluate the theoretical injection efficiency of hot electrons induced by the LSPs individually. Before 

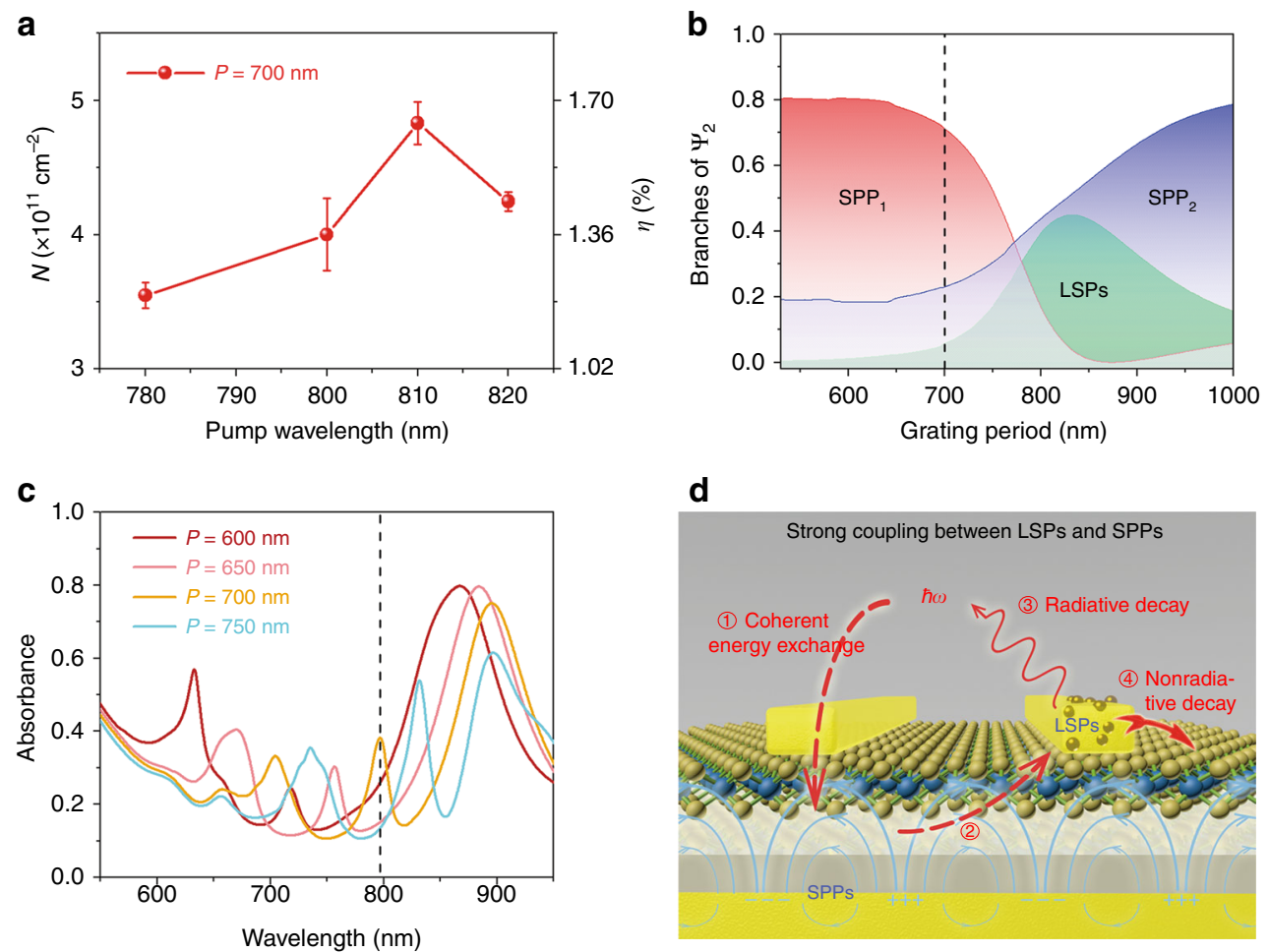

Fig. 4 Evidence for confirming the effect of strong coupling and the proposed model. a Transferred hot electron densities and corresponding $\eta$ for the heterostructure with a $700 \mathrm{~nm}$ grating period at different pump wavelengths. $\mathbf{b}$ Branches of $\Psi_{2}\left(\left|c_{21}\right|^{2},\left|c_{22}\right|^{2}\right.$, and $\left.\left|c_{23}\right|^{2}\right)$ as a function of the grating period. c The simulated absorbance of the heterostructures with grating periods of 600,650, 700, and $750 \mathrm{~nm}$. For a grating period of $700 \mathrm{~nm}$, the absorbance is approximately 0.4 when $\Psi_{2}$ is resonantly excited. $\mathbf{d}$ A diagrammatic model proposed to describe the physical processes in the strong coupling regime between LSPs and surface plasmon polaritons. Coherent energy exchange is represented by the dashed lines labeled by (1) and (2). The processes labeled as (3) and (4) denote the radiative and nonradiative relaxation channels of the LSPs, respectively. LSP localized surface plasmon

transferring into $\mathrm{MoS}_{2}$, many steps must occur for the LSPs to generate hot electrons. First, pump pulses couple to $\Psi_{2}$ with an absorbance of approximately $40 \%$ (Fig. 4c), and only $6 \%$ of the coupled energy is used to stimulate LSPs. In practice, both hot electrons and holes are excited, all of which are distributed in a range of energy and momentum. Assuming that hot electrons attain half of the energies and all are injected into $\mathrm{MoS}_{2}$, the efficiency is $1.2 \%(=40 \% \times 6 \% \times 50 \%)$ when considering the steps up to now, which equals the measured yield when pumped at $780 \mathrm{~nm}$. However, in fact, only energetic hot electrons with enough vertical momentum are capable of crossing the Schottky barrier ${ }^{17,29}$. In addition, they must overcome interfacial reflection and recombination with defects at the interface before finally transferring into $\mathrm{MoS}_{2}$. This comparison result suggests that $\eta$ should much less than the measured value if hot electrons are solely produced by the LSPs.

Based on the analysis above, we propose a model to depict the mechanism as illustrated in Fig. 4d, in which strong coupling between plasmonic modes is the ultimate reason for the elevated $\eta$. In the strong coupling regime, the energies of uncoupled oscillators are coherently exchanging $^{31}$. In this case, photons emitted by the radiative damping of LSPs are reabsorbed into SPPs by coherent energy exchange (1). As uncoupled SPPs with prolonged lifetimes are almost relaxed nonradiatively, their captured energies can be stored for a relatively long time. Thereafter, the intrinsic energies of the SPPs and reabsorbed energies can also be delivered to the LSPs through coherent energy exchange (2), experiencing radiative (3) and nonradiative (4) relaxations once again. The net result is that the original energies stored in the SPPs and radiation energies of the LSPs are recycled to produce hot carriers rather than being exhausted as heat or radiating into free space directly, such that the inaccessible energies in the SPPs are utilized and radiative damping in the LSPs is suppressed. In the strong coupling regime, the enhanced electric field surrounding $\mathrm{MoS}_{2}$ at resonance is another element that facilitates hot electron transfer. The electric field induced by image charges in the Au grating and Au film provides hot electrons with requisite vertical momenta for crossing the Schottky barrier. In such a picture, LSPs and SPPs collaborate excellently to take full advantage of unavailable energies for generating hot carriers in the strong coupling regime. 


\section{Discussion}

In summary, by virtue of femtosecond pump-probe spectroscopy, direct plasmonic hot electron transfer from an $\mathrm{Au}$ grating to an $\mathrm{MoS}_{2}$ monolayer was successfully observed in an MIM structure in the strong coupling regime. Plasmonic hot electron transfer occurs at $\sim 40 \mathrm{fs}$ with a maximum $\eta$ of $1.65 \%$. Strong coupling between LSPs and SPPs generates plasmonic hot electrons from energy and momentum. Coherent energy exchange allows photons radiated by the decay of LSPs to be reabsorbed by SPPs and originally unavailable energies stored in SPPs to participate in generating hot carriers. In this picture, low-loss SPPs that are nonradiative in nature function as an "energy recycle bin": capturing, storing and delivering radiative energies of the LSPs. Due to the intense electric field induced by image charges in the MIM structure, the perpendicular momenta required for crossing the Schottky barrier are provided. Ascribed to strong coupling, the complementary aspects of LSPs and SPPs overcome the intrinsic drawbacks of individual plasmonic modes in exploiting hot carriers. The insight presented in this work is also applicable to other metal-2D semiconductor, metal-molecular and metalorganic semiconductor systems. The spectral range of strong coupling can also be modulated in the near-infrared region by tuning the geometry and size, which holds great prospects for improving the performance of plasmonic hot carrier devices in fields involving photoconversion.

\section{Materials and methods}

\section{Sample preparations}

The $\mathrm{Au}$ film $(50 \mathrm{~nm})$ and $\mathrm{Al}_{2} \mathrm{O}_{3}$ layer $(20 \mathrm{~nm})$ were deposited on an $\mathrm{Si} / \mathrm{SiO}_{2}$ wafer by electron beam evaporation sequentially. The CVD-grown $\mathrm{MoS}_{2}$ monolayer was then transferred onto the prepared substrate with the wetting transfer method. The $\mathrm{MoS}_{2}$ monolayer grown on an $\mathrm{Si} / \mathrm{SiO}_{2}$ wafer was first spin-coated with poly(methyl methacrylate) (PMMA) and baked on a hot plate $\left(180{ }^{\circ} \mathrm{C}\right)$ for $5 \mathrm{~min}$. It was then etched in $\mathrm{KOH}$ solution for $6 \mathrm{~h}$ to strip the film from the wafer. Next, the film was removed and transferred to deionized water to rinse off impurities. This cleaning process was repeated three times. The film was then transferred onto the prepared substrate and heated for $5 \mathrm{~min}$ on a $100{ }^{\circ} \mathrm{C}$ hot plate. The PMMA on the film was dissolved with acetone steam that was produced by heating acetone at $150{ }^{\circ} \mathrm{C}$. An $\mathrm{MoS}_{2}$ monolayer was eventually left on the substrate. Au gratings $40 \mathrm{~nm}$ in thickness and $85 \pm 5 \mathrm{~nm}$ in width were fabricated using electron beam lithography and electron beam evaporation methods. To obtain a cross-sectional SEM image, the heterostructure was etched by a focused ion beam.

\section{Reflectance measurements}

A hyperspectral imaging system (Cytoviva HISV3) was adapted to perform the reflectance measurements.
Reflectance spectra were recorded by the spectrometer (Horiba iHR550) with a $\times 10$ objective (Olympus MPlanFL, NA $=0.25$ ). Relative reflectivity was utilized to represent the reflectance spectra, which was obtained by dividing the reflected intensity of the sample by that of the substrate. Nonpolarized white light passed through a linear polarizer (Thorlabs LPNIRE100-B) with the polarization direction along the $x$-axis. As the normal incident white light was confined by the objective, the in-plane wavevector $k_{x}$ was not zero despite the value being small compared with the momentum provided by gratings. However, this issue could not be neglected as it resulted in a splitting of uncoupled SPP modes $\left(\mathrm{SPP}_{1}\right.$ and $\left.\mathrm{SPP}_{2}\right)$.

\section{Finite-difference time-domain (FDTD) simulations}

The FDTD method was employed to simulate the reflectance spectra and electromagnetic field distributions of heterostructures. The relative permittivities of the $\mathrm{Au}$ and $\mathrm{MoS}_{2}$ monolayers were taken from the literature ${ }^{45,46}$, and the refractive index of $\mathrm{Al}_{2} \mathrm{O}_{3}$ was taken as 1.77. The incident angle of TM-polarized light was set at $3^{\circ}$ because oblique incidence with an angle of $\theta \sim 3^{\circ}$ can supply a similar in-plane wavevector to simulate the influence of objective confinement. Bloch boundary conditions were used in the $x$ direction, symmetric boundaries were applied in the $y$ direction and perfectly matched layer boundaries were used in the $z$ direction.

\section{Femtosecond pump-probe measurements}

Typical femtosecond transient absorption spectra measurements in a reflection configuration were carried out. The mode-locked oscillator (Tsunami 3941C-25XP) generated $800 \mathrm{~nm}$ femtosecond pulses with a repetition rate of $80 \mathrm{MHz}$ and a pulse duration of $\sim 73 \mathrm{fs}$. The output laser was split into two parts. One part was used as the pump and chopped at $1500 \mathrm{~Hz}$, while the other part was focused onto photonic crystal fiber (Newport SCG-800) to generate supercontinuum white light extending from 550 to $1400 \mathrm{~nm}$. The probe was then selected with a $650 \pm 10 \mathrm{~nm}$ bandpass filter (Thorlabs). The pump pulses passed through a linear polarizer (Thorlabs LPNIRE100-B), with the polarization direction along the $x$ axis. The reflected probe pulses were collected by a high-sensitivity photomultiplier (Thorlabs PMM02) and were converted into electric signals. The spot size of the focused probe was approximately $2 \mu \mathrm{m}$. The delay time between the pump and probe pulses was controlled by a stepper linear stage (Newport M-ILS150PP). Differential reflection signals $\Delta R / R_{0}(t)=\left(R-R_{0}\right) / R_{0}$ were acquired by subtracting the reflectivity of the probe with pump pulses $(R)$ from that without the pump $\left(R_{0}\right)$.

\section{Acknowledgements}

This work was supported by the National Key Research and Development Program of China (Grant No. 2017YFA0205700), National Basic Research 
Program of China (Grant Nos. 2015CB932403, 2017YFA0206000), National Science Foundation of China (Grant Nos. 11674012, 61422501, 11374023, 61521004 and 21790364), Beijing Natural Science Foundation (Grant No. L140007), Foundation for the Author of National Excellent Doctoral Dissertation of PR China (Grant No. 201420), National Program for Support of Top-notch Young Professionals (Grant No. W02070003) and Ministry of Education Singapore under Grant No. MOE2015-T2-2-043.

\section{Author details}

${ }^{1}$ School of Physics, State Key Lab for Mesoscopic Physics; Academy for Advanced Interdisciplinary Studies; Collaborative Innovation Center of Quantum Matter, Peking University, 100871 Beijing, China. ${ }^{2}$ CNRS InternationalNTU-Thales Research Alliance (CINTRA), Nanyang Technological University, Singapore 637553, Singapore. ${ }^{3}$ Centre for Micro-/Nano-Electronics (NOVITAS), School of Electrical and Electronic Engineering; Centre for Programmed Materials, School of Materials Science and Engineering, Nanyang Technological University, Singapore 637553, Singapore

\section{Authors' contributions}

Z.F. supervised the project. Y.Y. and H.S. performed the experiments. H.S. and Y. Y. established the theoretical model and carried out the simulations. All authors discussed the results and wrote the manuscript.

\section{Conflict of interest}

The authors declare that they have no conflict of interest.

Supplementary information is available for this paper at https://doi.org/ 10.1038/s41377-019-0121-6.

Received: 15 June 2018 Revised: 3 January 2019 Accepted: 3 January 2019 Published online: 16 January 2019

\section{References}

1. Barnes, W. L., Dereux, A. \& Ebbesen, T. W. Surface plasmon subwavelength optics. Nature 424, 824-830 (2003).

2. Brongersma, M. L., Halas, N. J. \& Nordlander, P. Plasmon-induced hot carrier science and technology. Nat. Nanotechnol. 10, 25-34 (2015).

3. Clavero, C. Plasmon-induced hot-electron generation at nanoparticle/metaloxide interfaces for photovoltaic and photocatalytic devices. Nat. Photonics $\mathbf{8}$, 95-103 (2014).

4. Knight, M. W., Sobhani, H., Nordlander, P. \& Halas, N. J. Photodetection with active optical antennas. Science 332, 702-704 (2011).

5. Zhong, J. H. et al. Probing the electronic and catalytic properties of a bimetallic surface with $3 \mathrm{~nm}$ resolution. Nat. Nanotechnol. 12, 132-136 (2016).

6. Kim, S. et al. High-harmonic generation by resonant plasmon field enhancement. Nature 453, 757-760 (2008).

7. Kawata, S., Inouye, Y. \& Verma, P. Plasmonics for near-field nano-imaging and superlensing. Nat. Photonics 3, 388-394 (2009).

8. Wurtz, G. A. et al. Designed ultrafast optical nonlinearity in a plasmonic nanorod metamaterial enhanced by nonlocality. Nat. Nanotechnol. 6, 107-111 (2011).

9. Baida, H. et al. Ultrafast nonlinear optical response of a single gold nanorod near its surface plasmon resonance. Phys. Rev. Lett. 107, 057402 (2011).

10. Hartland, G. V. Optical studies of dynamics in noble metal nanostructures. Chem. Rev. 111, 3858-3887 (2011).

11. Manjavacas, A., Liu, J. G., Kulkarni, V. \& Nordlander, P. Plasmon-induced hot carriers in metallic nanoparticles. ACS Nano 8, 7630-7638 (2014).

12. Tan, S. J. et al. Plasmonic coupling at a metal/semiconductor interface. Nat. Photonics 11, 806-812 (2017).

13. Giugni, A. et al. Hot-electron nanoscopy using adiabatic compression of surface plasmons. Nat. Nanotechnol. 8, 845-852 (2013).

14. Wu, K., Chen, J., McBride, J. R. \& Lian, T. Efficient hot-electron transfer by a plasmon-induced interfacial charge-transfer transition. Science 349, 632-635 (2015).

15. Harutyunyan, $\mathrm{H}$. et al. Anomalous ultrafast dynamics of hot plasmonic electrons in nanostructures with hot spots. Nat. Nanotechnol. 10, 770-774 (2015).
16. Furube, A., Du, L. C., Hara, K., Katoh, R. \& Tachiya, M. Ultrafast plasmon-induced electron transfer from gold nanodots into $\mathrm{TiO}_{2}$ nanoparticles. J. Am. Chem. Soc 129, 14852-12853 (2007).

17. Li, W. \& Valentine, J. G. Harvesting the loss: surface plasmon-based hot electron photodetection. Nanophotonics 6, 177-191 (2017).

18. Yu, Y. et al. Ultrafast plasmonic hot electron transfer in Au nanoantenna/MoS heterostructures. Adv. Funct. Mater. 26, 6394-6401 (2016).

19. Kang, Y. M. et al. Plasmonic hot electron induced structural phase transition in a MoS 2 monolayer. Adv. Mater. 26, 6467-6471 (2014).

20. Hoang, C. V. et al. Interplay of hot electrons from localized and propagating plasmons. Nat. Commun. 8, 771 (2017).

21. Kim, M., Lin, M. H., Son, J., Xu, H. X. \& Nam, J. M. Hot-electron-mediated photochemical reactions: principles, recent advances, and challenges. Adv. Opt. Mater. 5, 1700004 (2017).

22. Wen, X. L., Xu, W. G., Zhao, W. J., Khurgin, J. B. \& Xiong, Q. H. Plasmonic hot carriers-controlled second harmonic generation in $\mathrm{WSe}_{2}$ bilayers. Nano Lett. 18, 1686-1692 (2018).

23. Fang, Z. Y. et al. Graphene-antenna sandwich photodetector. Nano Lett. 12 3808-3813 (2012).

24. Sobhani, A. et al. Narrowband photodetection in the near-infrared with a plasmon-induced hot electron device. Nat. Commun. 4, 1643 (2013).

25. Li, W. \& Valentine, J. Metamaterial perfect absorber based hot electron photodetection. Nano Lett. 14, 3510-3514 (2014).

26. Li, W. et al. Circularly polarized light detection with hot electrons in chiral plasmonic metamaterials. Nat. Commun. 6, 8379 (2015).

27. Sundararaman, R. et al. Theoretical predictions for hot-carrier generation from surface plasmon decay. Nat. Commun. 5, 5788 (2014).

28. Bernardi, M., Mustafa, J., Neaton, J. B. \& Louie, S. G. Theory and computation of hot carriers generated by surface plasmon polaritons in noble metals. Nat. Commun. 6, 7044 (2015)

29. Narang, P., Sundararaman, R. \& Atwater, H. A. Plasmonic hot carrier dynamics in solid-state and chemical systems for energy conversion. Nanophotonics $\mathbf{5}$ 96-111 (2016).

30. Törmä, P. \& Barnes, W. L. Strong coupling between surface plasmon polaritons and emitters: a review. Rep. Prog. Phys. 78, 013901 (2015).

31. Vasa, P. et al. Real-time observation of ultrafast Rabi oscillations between excitons and plasmons in metal nanostructures with J-aggregates. Nat. Photonics 7, 128-132 (2013).

32. Groß, H., Hamm, J. M., Tufarelli, T., Hess, O. \& Hecht, B. Near-field strong coupling of single quantum dots. Sci. Adv. 4, eaar4906 (2018).

33. Kleemann, M.E. et al. Strong-coupling of $\mathrm{WSe}_{2}$ in ultra-compact plasmonic nanocavities at room temperature. Nat. Commun. 8, 1296 (2017).

34. Thomas, R. et al. Plexcitons: the role of oscillator strengths and spectral widths in determining strong coupling. ACS Nano 12, 402-415 (2018).

35. Chen, H. J. et al. Plasmon-molecule interactions. Nano Today 5, 494-505 (2010).

36. Zheng, D. et al. Manipulating coherent plasmon-exciton interaction in a single silver nanorod on monolayer WSe 2 . Nano Lett. 17, 3809-3814 (2017).

37. Zeng, P. et al. Photoinduced electron transfer in the strong coupling regime: waveguide-plasmon polaritons. Nano Lett. 16, 2651-2656 (2016).

38. Rodriguez, S. R. K., Murai, S., Verschuuren, M. A. \& Rivas, J. G. Light-emitting waveguide-plasmon polaritons. Phys. Rev. Lett. 109, 166803 (2012).

39. Konrad, A., Kern, A. M., Brecht, M. \& Meixner, A. J. Strong and coherent coupling of a plasmonic nanoparticle to a subwavelength fabry-pérot resonator. Nano Lett. 15, 4423-4428 (2015).

40. Chu, Y. Z. \& Crozier, K. B. Experimental study of the interaction between localized and propagating surface plasmons. Opt. Lett. 34, 244-246 (2009).

41. Liu, W. J. et al. Strong exciton-plasmon coupling in $\mathrm{MoS}_{2}$ coupled with plasmonic lattice. Nano Lett. 16, 1262-1269 (2016).

42. Cade, N. I., Ritman-Meer, T. \& Richards, D. Strong coupling of localized plasmons and molecular excitons in nanostructured silver films. Phys. Rev. B 79, 241404 (2009).

43. Bellessa, J. et al. Exciton/plasmon polaritons in $\mathrm{GaA} \mathrm{Al} \mathrm{l}_{0.93} \mathrm{Ga}_{0.07} \mathrm{As}$ heterostructures near a metallic layer. Phys. Rev. B 78, 205326 (2008).

44. Su, M. N. et al. Optomechanics of single aluminum nanodisks. Nano Lett. 17 2575-2583 (2017)

45. Johnson, P. B. \& Christy, R. W. Optical constants of the noble Metals. Phys. Rev. B 6, 4370-4379 (1972).

46. Liu, J. T., Wang, T. B., Li, X. J. \& Liu, N. H. Enhanced absorption of monolayer $\mathrm{MoS}_{2}$ with resonant back reflector. J. Appl. Phys. 115, 193511 (2014). 